\title{
Three-Wheeled Omnidirectional Robot Localization in RFID-Tag Environments using UFIR Filtering
}

\author{
JORGE A. ORTEGA- \\ CONTRERAS \\ Dept. of Electronics \\ Engineering Universidad de \\ Guanajuato \\ Salamanca, MEXICO
}

\author{
YURIY S. SHMALIY \\ Dept. of Electronics \\ Engineering Universidad de \\ Guanajuato \\ Salamanca, MEXICO
}

\author{
JOSE A. ANDRADE-LUCIO \\ Dept. of Electronics \\ Engineering Universidad de \\ Guanajuato \\ Salamanca, MEXICO
}

\begin{abstract}
This paper describes a way to improve the indoor navigation of mobile robots using radio frequency identification (RFID) technology. A net of RFID tags is deployed in the navigation space. A measurement system measures distances from the tags to the robot with in the presence of the firstorder Markov-Gauss colored measurement noise (CMN) and is combined with a digital gyroscope to measure the robot heading. To increase the localization accuracy, the Kalman filter (KF) and unbiased finite impulse response (UFIR) modified for CMN are used. It is shown that the navigation system developed is more accurate than the basic one employing the standard KF and UFIR filter.
\end{abstract}

Key Words: Robot localization, colored measurement noise, Kalman Filter, UFIR filter

Received: August 23, 2020. Revised: December 10, 2020. Accepted: February 16, 2021. Published: February 28, 2021.

\section{Introduction}

Wireless technologies such as the radio frequency identification (RFID) tag-based systems have attracted the interest of many consumers due to low cost and low (or zero) energy consumption and a wide distance range that made them standard for indoor object navigation and tracking [1]-[8]. In practical designs of mobile robot navigation systems, one finds various efficient hybrid solutions, such as the localization scheme combining information available from the RFID tag-based networks and other sensors. In [9], a novel localization method is proposed to combine the RFID tag-based data with laserbased measurements. In [10], a variable power RFID model is proposed for the localization over passive ultra-high frequency (UHF) RFID tag nets in complex environments. In [11], a location system is designed to combines two types of the RFID tag-generated signals with a logical classification strategy and the integration is provided using the Bayesian filter-based algorithms (BFA). The objective of the BFA is to compute the posterior distributions of the states of a dynamic system, given an observation function with noise. This method has many advantages, but the more remarkable is the capability to represent a complex distribution without requiring information about the state-space model or the state distributions, although with a high computational cost.

The state estimation problem [12], [13] can be solved for linear Gaussian processes and observations using the Kalman Filter (KF) and for non-linear models using the Extended $\mathrm{KF}$ (EKF) or unscented KF (UKF). Another approach is the unbiased finite impulse response (UFIR) filter [14], which can also be applied to linear models and nonlinear models [15] as described in [16]. An advantage is that the UFIR algorithm does not require the noise statistics and has a better robustness.

Navigation over the RFID tag nets is typically provided in the presence of the colored measurement noise (CMN) [17]-[19]. To estimate the robot state under CMN, there can be used two well-known approaches developed by Bryson et al. in [20], [21] and Petovello et al. in [22]. In the Bryson algorithm, the CMN is filtered out in two phases: smoothing and filtering. In the Petovello algorithm, only one stage (filtering) is needed. Another solutions were found by Shmaliy et al. to deal with the colored process noise using state differencing [23], Zhou et al. by using the second moment of information [24], and Ding et al. by applying the least squaresbased iterative parameter estimation to dynamical systems with the autoregressive moving average (ARMA) noise model [25]. In this paper, we apply the KF and UFIR filter modified in [26] under CMN to provide an accurate robot navigation over RFID tag networks.

\section{Robot Dynamics and Modeling}

In this section, we consider the three-wheel omnidirectional robot dynamics and represent it is discrete-time state-space. An omnidirectional mobile robot pictured in Fig. 1 is often designed for plane motion to have three degrees of freedom (DOF). Depending on the types of dynamic constraints, a mobile wheeled robot can control each DOF independently. In this case, it is called non-holonomic and can travel in any direction without orientation restrictions.

\subsection{Dynamics of the Transition State Function}

A kinematic diagram of a three-wheeled omnidirectional robot (TWOR) with perpendicular rollers is shown in Fig. 2. The robot is driven by the displacement in its wheels. Assuming a no-slip condition, the displacement $\Delta u_{j}$ in each wheel can be found by projecting the displacement vector $u_{j}$ into the local coordinate system $\left(x_{L}, y_{L}\right)$ and using the contribution of the arc segment caused by the rotation $\Delta \varphi$ of the robot. That results in

$$
\Delta u_{j}=\left\|y{\overrightarrow{u_{j}}}_{x_{L}}\right\|+\left\|y{\overrightarrow{u_{j}}}_{y_{L}}\right\|+\Delta \varphi * R
$$

where $R$ is the radius of the robot platform. 


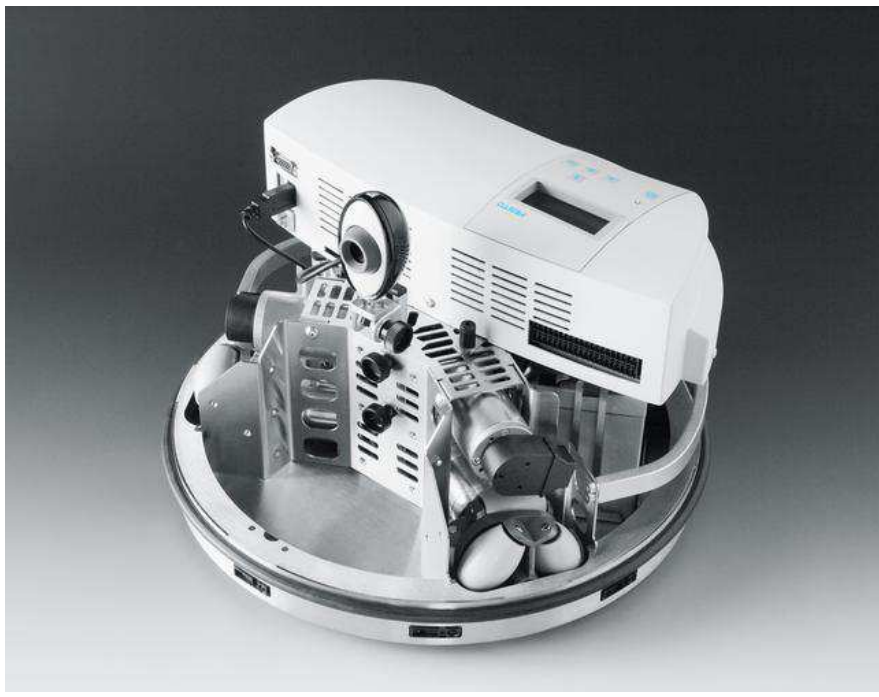

Fig. 1. Festo Robotino Mobile Robotic Development Platform

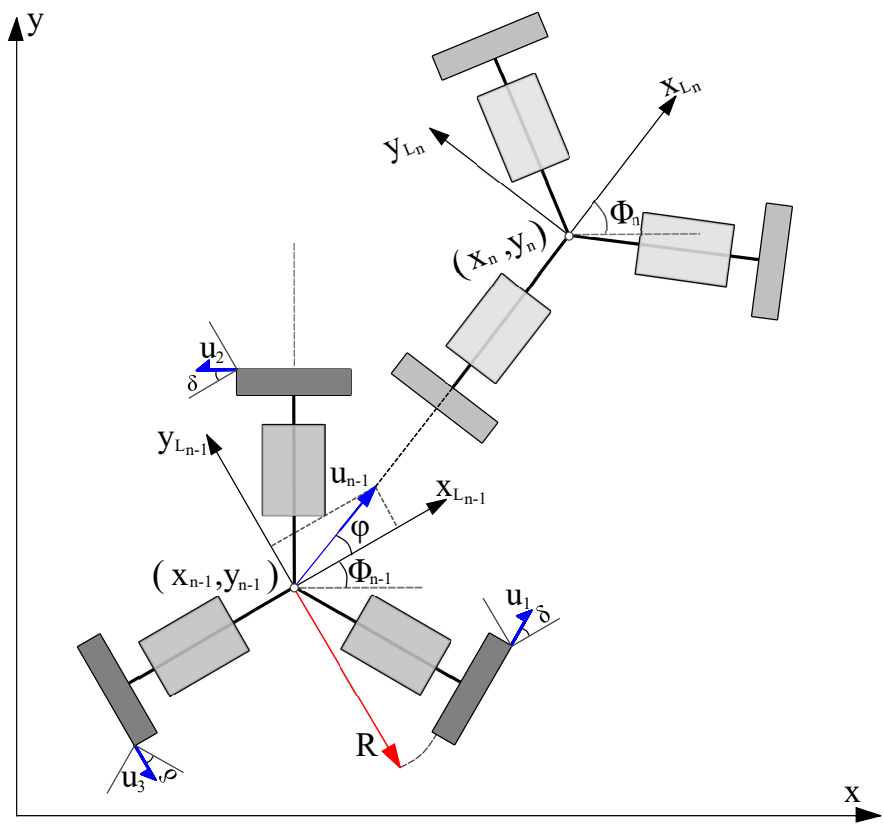

Fig. 2. Kinematic diagram of TWOR

From (1), the robot dynamics can be represented in state space with

$$
\begin{aligned}
{\left[\begin{array}{c}
\Delta u_{1} \\
\Delta u_{2} \\
\Delta u_{3}
\end{array}\right] } & =\left[\begin{array}{ccc}
\cos \delta & \sin \delta & R \\
-\cos \delta & \sin \delta & R \\
0 & -1 & R
\end{array}\right]\left[\begin{array}{c}
\Delta u_{x_{L}} \\
\Delta u_{y_{L}} \\
\Delta \varphi
\end{array}\right], \\
\Delta U_{i} & =\mathbf{A} \Delta U_{L}
\end{aligned}
$$

where $\Delta U_{i}$ is the displacements vector, vector $\Delta U_{L}$ contains information about variations in the robot position with respect to the local coordinates, and $\mathbf{A}$ is the system matrix. By inverting (2), it is possible to determine the increments in the local coordinates as functions of the wheels displacement $\Delta U_{i}$.
Since $\delta=\pi / 6$ in Fig. 2, we have

$$
\begin{aligned}
\Delta U_{L} & =A^{-1} \Delta U_{i} \\
A^{-1} & =\left[\begin{array}{ccc}
\sqrt{3} / 3 & -\sqrt{3} / 3 & 0 \\
1 / 3 & 1 / 3 & -2 / 3 \\
1 / 3 R & 1 / 3 R & 1 / 3 R
\end{array}\right] .
\end{aligned}
$$

To found the position in the global coordinate system we need to apply the matrix rotation operator $R(\Phi)$

$$
\begin{aligned}
& \Delta U=R(\Phi) U_{L} \\
& \Delta U=R(\Phi) A^{-1} U_{i}
\end{aligned}
$$

that allows finding the displacements as

$$
\begin{array}{r}
\Delta u_{x}=\frac{\sqrt{3}}{3} \Delta u_{12} \cos \left(\Phi_{n-1}\right)-\frac{1}{3} \Delta u_{123} \sin \left(\Phi_{n-1}\right), \\
\Delta u_{y}=\frac{\sqrt{3}}{3} \Delta u_{12} \sin \left(\Phi_{n-1}\right)+\frac{1}{3} \Delta u_{123} \cos \left(\Phi_{n-1}\right), \\
\Delta \varphi=\frac{1}{3 R}\left(\Delta u_{1}+\Delta u_{2}+\Delta u_{3}\right),
\end{array}
$$

where $\Delta u_{12}=\Delta u_{1}-\Delta u_{2}$ and $\Delta u_{123}=\Delta u_{1}+\Delta u_{2}-2 \Delta u_{3}$. By introducing the state vector $x_{n}=\left[\begin{array}{lll}x_{1 n} & x_{2 n} & x_{3 n}\end{array}\right]^{T}$ of the global coordinates, with the components

$$
\begin{aligned}
& x_{1_{n}}=x_{1_{n-1}}+\Delta u_{x}, \\
& x_{2_{n}}=x_{2_{n-1}}+\Delta u_{y}, \\
& x_{3_{n}}=x_{3_{n-1}}+\Delta \varphi,
\end{aligned}
$$

the nonlinear state equation can be written as

$$
x_{n}=f\left(x_{n-1}, u_{n}, w_{n}\right),
$$

where $w_{n} \sim \mathcal{N}\left(0, Q_{n}\right)$ is zero mean white Gaussian noise with the covariance $Q_{n}$.

\subsection{Nonlinear Observation}

Let us suppose that the RFID environment is organized with $k$-tags $T_{j}, j \in[1, k]$, having known coordinates $\left(\chi_{j}, \mu_{j}, \nu_{j}\right)$ and at least three tags are available at each $n$. The robot position can be computed via distances measured between the tags and the robot. The robot heading in global coordinates is measured directly by a digital gyroscope and the observation equations thus become

$$
\begin{aligned}
d_{1 n} & =\sqrt{\left(x_{1 n}-\chi_{1}\right)^{2}+\left(x_{2 n}-\mu_{1}\right)^{2}+\nu_{1}^{2}}+v_{1 n}, \\
d_{2 n} & =\sqrt{\left(x_{1 n}-\chi_{2}\right)^{2}+\left(x_{2 n}-\mu_{2}\right)^{2}+\nu_{2}^{2}}+v_{2 n}, \\
\vdots & \\
d_{k n} & =\sqrt{\left(x_{1 n}-\chi_{k}\right)^{2}+\left(x_{2 n}-\mu_{k}\right)^{2}+\nu_{k}^{2}}+v_{k n}, \\
\Phi_{n} & =\phi_{n}+v_{\Phi n}
\end{aligned}
$$

where the $j$ th, $j \in[1, k]$, Markov-Gauss noise vector is modeled as

$$
v_{j n}=\Psi_{j n} v_{j(n-1)}+\xi_{j n}
$$

where $\xi_{j n} \sim \mathcal{N}\left(0, R_{j n}\right)$ is zero mean white Gaussian driving noise with the covariance $R_{j n}$ and $0<\Psi_{j n}<1$ is the coloredness factor, which zero value, $\Psi_{j n}=0$ makes noise $v_{j n}$ white Gaussian. 


\subsection{Extended Space-state Model}

To apply UFIR filtering to nonlinear models, the first-order Taylor series expansion can be applied to the state equation

(9) that results in the following extended state equation [15]

$$
\begin{aligned}
x_{n} & =F_{n} x_{n-1}+\left[f_{n}\left(\hat{x}_{n-1}, u_{n}, 0\right)-F_{n} \hat{x}_{n-1}\right]+L_{n} w_{n} \\
& =F_{n} x_{n-1}+\tilde{u}_{n}+\tilde{w}_{n},
\end{aligned}
$$

where the Jacobian matrices $F_{n}$ and $L_{n}$ and vectors $\tilde{u}_{n}$ and $\tilde{w}_{n}$ are defined as

$$
\begin{aligned}
F_{n} & =\left.\frac{\partial f_{n}}{\partial x}\right|_{\hat{x}_{n-1}} \\
L_{n} & =\left.\frac{\partial f_{n}}{\partial w}\right|_{\hat{x}_{n-1}} \\
\tilde{u}_{n} & =f_{n-1}\left(\hat{x}_{n-1}, u_{n}, 0\right)-F_{n} \hat{x}_{n-1} \\
\tilde{w}_{n} & \sim \mathcal{N}\left(0, L_{n} Q_{n} L_{n}^{T}\right) .
\end{aligned}
$$

Similarly, the observation (10) can be described with

$$
\begin{gathered}
y_{n}=h\left(x_{n}, v_{n}\right) \\
v_{n}=\Psi_{n} v_{n-1}+\xi_{n} \\
\xi_{n} \sim \mathcal{N}\left(0, R_{n}\right),
\end{gathered}
$$

where $y_{n}=\left[\begin{array}{lllll}y_{1 n} & y_{2 n} & \ldots & y_{k n} & y_{\Phi n}\end{array}\right]^{T} \in \mathbb{R}^{k+1}$ is the observation vector, $h\left(x_{n}, v_{n}\right)$ is a nonlinear function, and $v_{n}$ is the CMN modeled as the Markov-Gauss process.

For additive noise $v_{n}$, the nonlinear observation equation can be extended by the first-order Taylor series as

$$
\begin{aligned}
y_{n} & =h\left(\hat{x}_{n}^{-}, 0\right)+\left.\frac{\partial h_{n}}{\partial x}\right|_{\hat{x}_{n}^{-}}\left(x_{n}-\hat{x}_{n}^{-}\right)+\frac{\partial h_{n}}{\partial v} v_{n} \\
& =h\left(\hat{x}_{n}^{-}, 0\right)+H_{n}\left(x_{n}-\hat{x}_{n}^{-}\right)+M_{n} v_{n} \\
& =H_{n} x_{n}+z_{n}+\tilde{v}_{n},
\end{aligned}
$$

where $\hat{x}_{n}^{-} \triangleq \hat{x}_{n \mid n-1}=F_{n} \hat{x}_{n-1 \mid n-1}$ is the prior estimate at $n$. The Jacobian matrices $H_{n}$ and $M_{n}$ and vectors $\tilde{v}_{n}$ and $z_{n}$ are defined by

$$
\begin{aligned}
H_{n} & =\left.\frac{\partial h_{n}}{\partial x}\right|_{\hat{x}_{n}^{-}} \\
M_{n} & =\left.\frac{\partial h_{n}}{\partial v}\right|_{\hat{x}_{n}^{-}} \\
z_{n} & =h\left(\hat{x}_{n}^{-}, 0\right)-H_{n} \tilde{x}_{n}^{-} \\
\tilde{v}_{n} & \sim \mathcal{N}\left(0, M_{n} R_{n} M_{n}^{T}\right)
\end{aligned}
$$

Using the extended state and observation equations, the UFIR filter can be modified for CMN as follows.

\subsection{Model Modification for CMN}

By introducing a new observation $z_{n}$ using measurement differencing and following [26], we obtain

$$
\begin{aligned}
z_{n} & =y_{n}-\Psi_{n} y_{n-1} \\
& =H_{n} x_{n}+v_{n}-\Psi_{n} H_{n-1} x_{n-1}-\Psi_{n} v_{n-1}
\end{aligned}
$$

Using the extended state-space model we also provide

$$
z_{n}=D_{n} x_{n}+\bar{v}_{n},
$$

where $\bar{v}_{n}$ is white noise with the covariance

$$
\begin{gathered}
E\left\{\bar{v}_{n} \bar{v}_{n}^{T}\right\}=\Gamma_{n} \Phi_{n}+R_{n}, \\
E\left\{\bar{v}_{n} w_{n}^{T}\right\}=\Gamma_{n} L_{n} Q_{n}, \\
\Phi_{n}=L_{n} Q_{n} L_{n}^{T} \Gamma_{n}^{T},
\end{gathered}
$$

and $D_{n}, \Gamma_{n}$, and $\bar{v}_{n}$ are give by

$$
\begin{array}{r}
\Gamma_{n}=\Psi_{n} H_{n-1} F_{n}^{-1}, \\
D_{n}=H_{n}-\Gamma_{n}, \\
\bar{v}_{n}=\Gamma_{n} L_{n} w_{n}+\xi_{n} .
\end{array}
$$

It follows that noise $\bar{v}_{n}$ is time-correlated with $w_{n}$. Using the above provided extensions, the filtering algorithms can be modified as follows.

\subsection{Kalman Filter for CMN}

Provided a modified observation model (14) with timecorrelated $w_{n}$ and $\bar{v}_{n}$, a new Kalman gain is required by minimizing the trace of the error covariance $P_{n}$ in order for the KF to be optimal. To find $P_{n}$, the prior error covariance can be defined as

$$
P_{n}^{-}=E\left\{\left(x_{n}-\hat{x}_{n}^{-}\right)\left(x_{n}-\hat{x}_{n}^{-}\right)^{T}\right\},
$$

the measurement residual $s_{n}$ and innovation covariance $S_{n}$ as

$$
\begin{aligned}
& s_{n}=z_{n}-D_{n} \hat{x}_{n}^{-}, \\
& S_{n}=E\left\{s_{n} s_{n}^{T}\right\},
\end{aligned}
$$

and then the $\mathrm{KF}$ estimate depicted as $\mathrm{CKF}$ for $\mathrm{CMN}$ as

$$
\hat{x}_{n}=\hat{x}_{n}^{-}+K_{n} s_{n} .
$$

By introducing the estimation error $\epsilon=x_{n}-\hat{x}_{n}$, the error covariance $P_{n}=E\left\{\epsilon_{n} \epsilon_{n}^{T}\right\}$ must then be transformed using the above definitions and its trace depicted as $\operatorname{tr}\left(P_{n}\right)$ minimized by the optimal cKF gain $K_{n}$. A detailed description of this procedure can be found in [26] and a pseudo-code for the $\mathrm{cKF}$ algorithm listed as Algorithm 1.

\subsection{UFIR Filter for CMN}

One of the advantages of the UFIR filter that was originally derived in [27] is that it does not need information about the noise statistics. Instead, it requires an optimal averaging horizon of $N_{\text {opt }}$ points to achieve the minimization of the mean square error (MSE). The algorithm requires an initialization on a short initial horizon over a data vector $Y_{m, s}=\left[y_{m} \cdots y_{s}\right]^{T}$, where $s=m+K-1$ and $K$ is the number of the states, and the generalized noise power gain (GNPG) $G_{s}$ to avoid singularities. Another advantage is that the UFIR filter ignores zero mean noise, so that the time-correlated zero mean and white $w_{n}$ and $\bar{v}_{n}$ can be discarded. A pseudo-code for the UFIR filter for CMN is listed in Algorithm 2. 

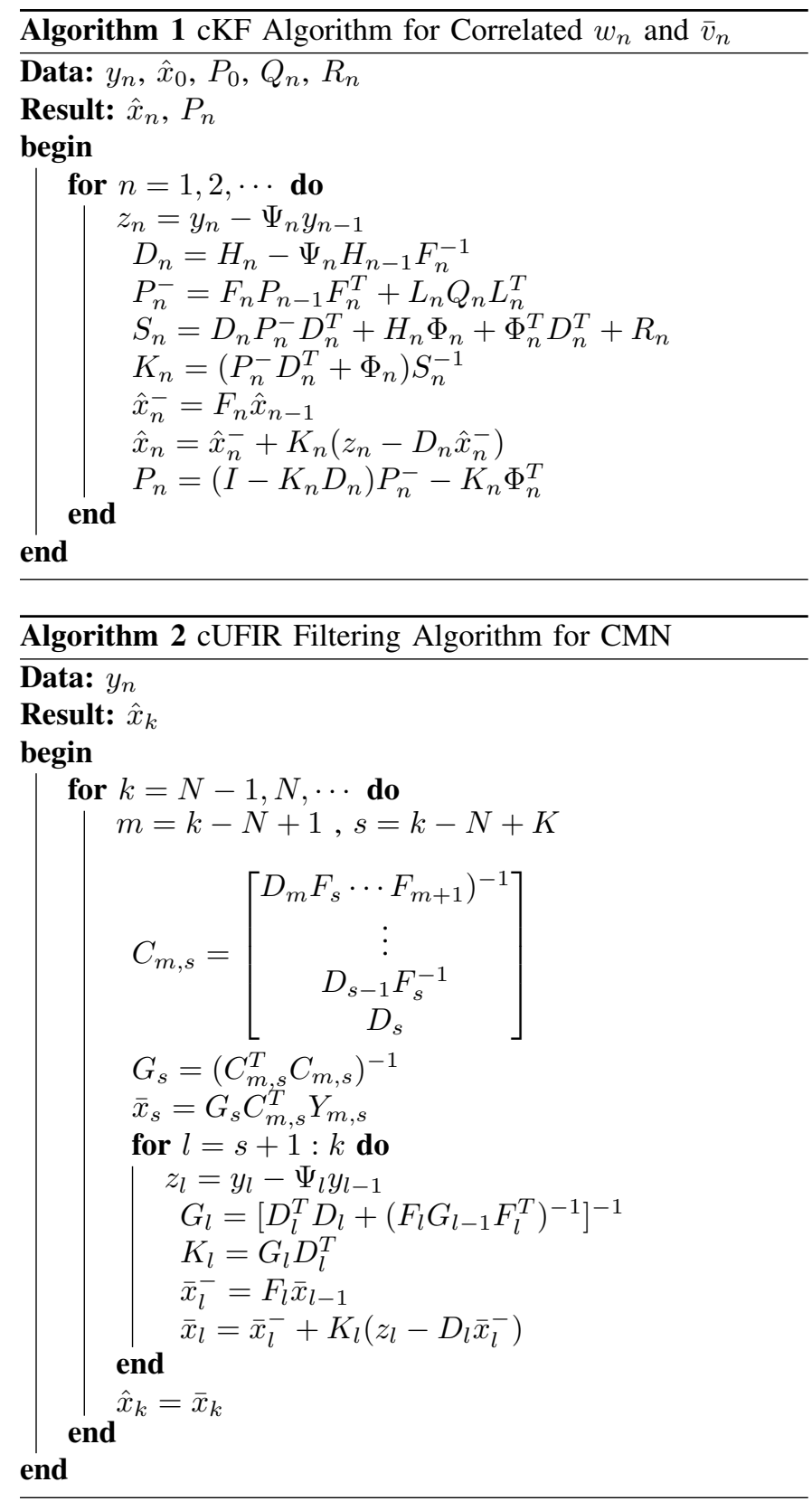

To optimize the the number of points $N$ as $N_{\text {opt }}$ in the MSE sense [28], the error covariance of the UFIR filter can be written as

$$
P_{n}=P_{n}^{-}\left(2 P_{n}^{-} D_{n}^{T}+2 \Phi_{n}+G_{n} D_{n}^{T} S_{n}\right) D_{n} G_{n}
$$

and then $N_{\text {opt }}$ found by minimizing $\operatorname{tr}\left(P_{n}\right)$ using the recursive equation (25) or in some other way as shown in [28].

\section{Omnidirectional Robot Tracking}

We now apply the cKF and cUFIR filter to estimate the state of the TWOR in two scenarios. In the first scenario, the TWOR travels circularly with a constant motor displacement as shown in Fig. 3 In the second scenario, we use experimental data available from [29]. The noise standard deviations are set

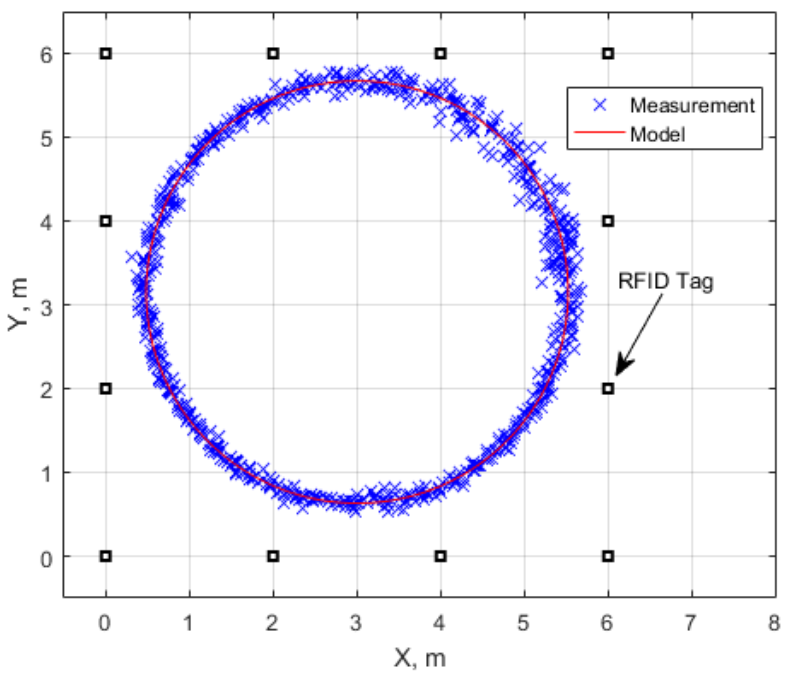

Fig. 3. A circular TWOR trajectory and noisy measurements.

as $\sigma_{w}=10 \mathrm{~cm}, \sigma_{v}=20 \mathrm{~cm}$, and $\sigma_{\Phi}=0.5^{\circ}$. The TWOR platform radius is $R=0.18 \mathrm{~m}$.

In the first test, the indoor navigation space is nested with 12 RFID tags, deployed on the perimeter with a maximum distance of $8 \mathrm{~m}$. All of the tags are visible from any point in the space as shown in Fig. 3. The coloredness factor $\Psi$ is changed from zero to 0.9 with a steps of 0.05 . Each simulation is repeated 100 times and the localization root MSEs (RMSEs) produced by the EKF, EFIR filter, cEKF, and cEFIR filter in the $x$ direction are sketched in Fig. 4 The RMSEs produced

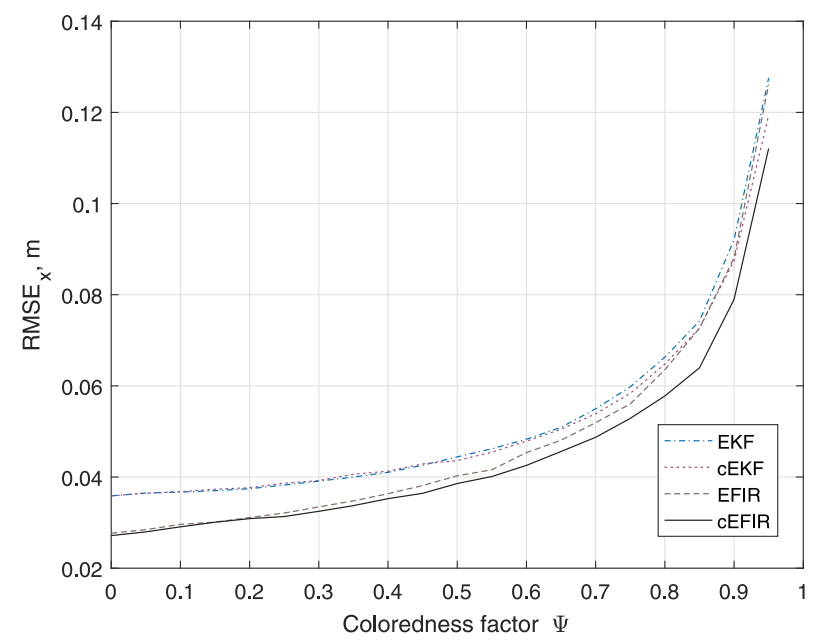

Fig. 4. RMSEs produced by the EKF, EFIR filter, cEKF, and cEFIR filter in the $\mathrm{x}$ direction.

in the y direction are shown in Fig. 5. As expected, errors sketched in the Fig. 4 and Fig. 5 grow with an increase in coloredness. It can also be seen that, by $\Psi=0$, the EKF and $\mathrm{CEKF}$ produce equal estimates and so do the EFIR and cEFIR filters. In both cases, the EFIR filter outperforms the EKF and cEKF and the cEFIR filter produces smallest errors among oll other filters. When $\Psi$ ranges lower than 0.5 , the 


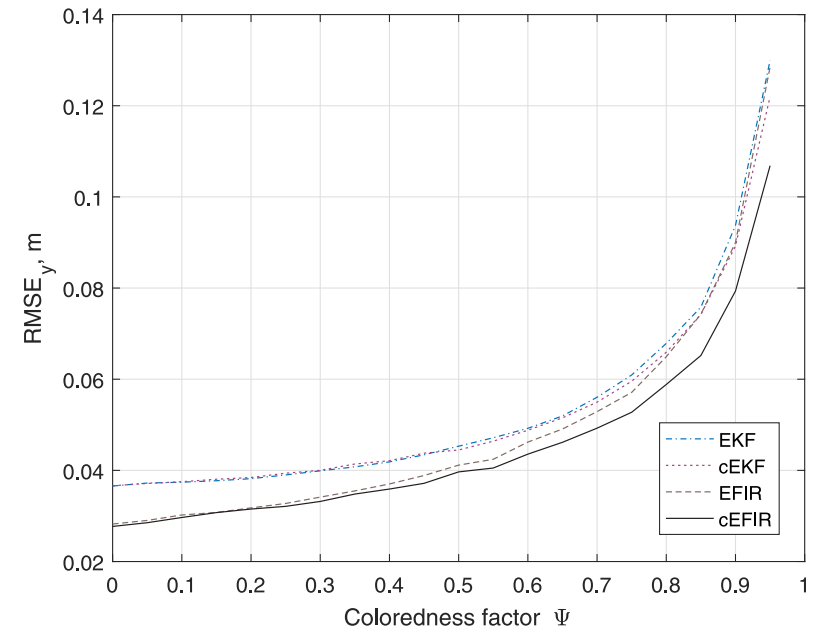

Fig. 5. RMSEs produced by the EKF, EFIR filter, cEKF, and cEFIR filter in the $\mathrm{y}$ direction.

pairs of KF and FIR estimates demonstrate no significative differences. However, larger values of $\Psi$ make the estimate different. Furthermore, errors in the EKF and EFIR filter grows more rapidly than in the modified versions.

In the second test, the trajectory of a mobile robot is represented with experimental data and the measurement vector created by the nonlinear observation function over 36 RFID tags [29]. The noisy measurement vector (for $\Psi_{n}=0$ ) and reference path are showed in Fig. 6. The RMSEs produced

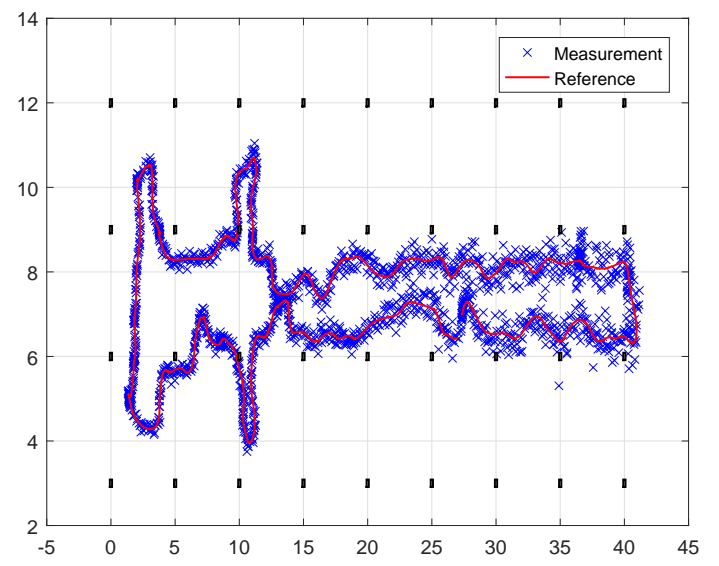

Fig. 6. Mobile robot measured trajectory and a reference path.

by the filters in the second test are sketched in Fig. 7 and Fig. 8. Overall, we watch here for the same error behaviours, although the cEFIR filter demonstrates dramatically smaller errors in the y direction.

\section{Conclusions}

Estimation of the mobile robot coordinates has been provided in this paper under the CMN using the EKF, cEKF, EFIR filter, and cEFIR filter. The CMN was modeled as

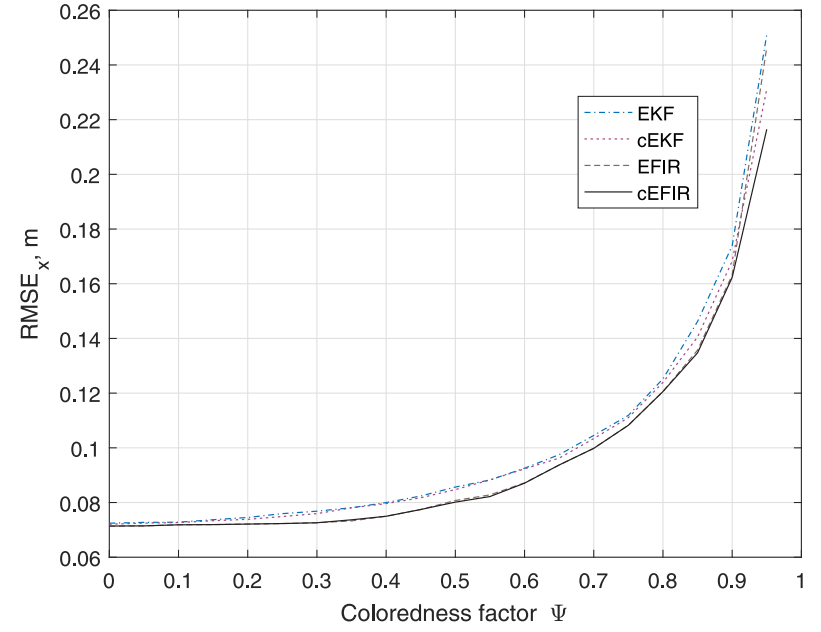

Fig. 7. RMSEs produced by the filters in the second test in the $\mathrm{x}$ direction.

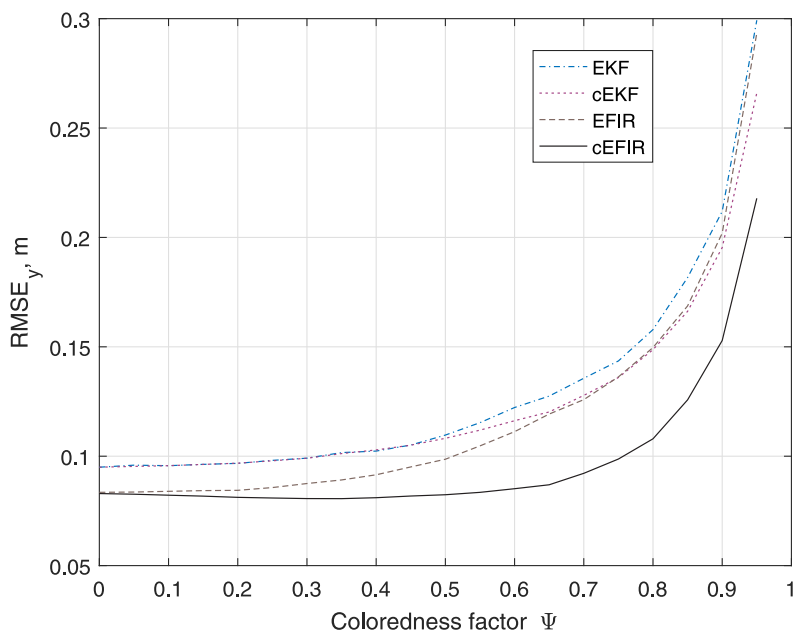

Fig. 8. RMSEs produced by the filters in the second test in the y direction.

Markov-Gauss and the coloredness factor set experimentally to minimize the MSE. It has been shown that the cEFIR outperforms other filters in both scenarios of a simulated circular trajectory and experimentally measured data and that $\mathrm{cKF}$ and cUFIR algorithms improve the RMSE with an increase in the coloredness factor. We also notice that further increase in the accuracy can be achieved by considering other distributions in the RFID tag signals [30]-[32].

\section{References}

[1] R. Zhao, Y. Zhang, G. Wang, and D. Wang, "Mobile robot localization using rotating synthetic aperture rfid," in 2018 IEEE CSAA Guidance, Navigation and Control Conference (CGNCC), 2018, pp. 1-6.

[2] X. Liu, J. Zhang, S. Jiang, Y. Yang, K. Li, J. Cao, and J. Liu, "Accurate localization of tagged objects using mobile RFID-augmented robots," IEEE Trans. Mobile Comput.,2019

[3] F. Martinelli, "Simultaneous localization and mapping using the phase of passive uhf-rfid signals," J. Intel. Robotic Syst., vol. 94, no. 34, pp. 711-725, Jul 2018.

[4] J. Chai, C. Wu, C. Zhao, H.-L. Chi, X. Wang, B. W.-K. Ling, and K. L. Teo, "Reference tag supported rfid tracking using robust support vector regression and kalman filter," Advanc. Eng. Informat., vol. 32 , pp. 110, Apr 2017. 
[5] J. Su, Z. Sheng, V. C. M. Leung, and Y. Chen, "Energy efficient tag identification algorithms for rfid: Survey, motivation and new design," IEEE Wirel. Comm., vol. 26, no. 3, pp. 118-124, 2019.

[6] M. P. Jayakrishnan, A. Vena, B. Sorli, and E. Perret, "Solid-state conductive-bridging reconfigurable rf-encoding particle for chipless rfid applications," IEEE Micr. Wirel. Compon. Lett., vol. 28, no. 6, pp. 506508, 2018.

[7] R. Malekian, A. F. Kavishe, B. T. Maharaj, P. K. Gupta, G. Singh, and H. Waschefort, "Smart vehicle navigation system using hidden markov model and RFID technology," Wirel. Person. Comm., vol. 90, no. 4, pp. 1717-1742, Jun. 2016

[8] P. Agarwal, A. Gupta, G. Verma, H. Verma, A. Sharma, and S. Banarwal, "Wireless monitoring and indoor navigation of a mobile robot using RFID," in Nature Inspired Computing, Springer Singapore, Oct. 2017, pp. 83-90.

[9] H. Wu, X. Wu, and G. Tian, "Indoor robot localization based on single rfid tag," Artificial Life and Robotics, vol. 23, no. 3, pp. 373-379, 2018.

[10] J. Zhang, Y. Lyu, J. Patton, S. C. G. Periaswamy, and T. Roppel, “A probabilistic UHF RFID tag localization algorithm using Bayesian filter and a variable power RFID model," IEEE Trans. Ind. Electron., vol. 65 , no. 10, pp. 8250-8259, 2018.

[11] B. Tao, H. Wu, Z. Gong, Z. Yin, and H. Ding, "An RFID-based mobile robot localization method combining phase difference and readability," IEEE Trans. Autom. Sci. Eng., 2019

[12] D. Simon, "Optimal state estimation: Kalman, $H_{\infty}$, and nonlinear approaches," Wiley, 2006.

[13] X. Zhang, F. Ding, and E. Yang, "State estimation for bilinear systems through minimizing the covariance matrix of the state estimation errors," Int. J. Adapt. Contr. Signal Process., vol. 33, no. 7, pp. 11571173, 2019.

[14] Y. S. Shmaliy, S. Zhao, and C. K. Ahn, "Unbiased finite impluse response filtering: an iterative alternative to Kalman filtering ignoring noise and initial conditions," IEEE Contr. Syst. Mag., vol. 37, no.5, pp. 70-89, 2017.

[15] Y. S. Shmaliy, "Suboptimal FIR filtering of nonlinear models in additive white Gaussian noise," IEEE Trans. Signal Process., vol. 60, no. 10, pp. 5519-5527, 2012.

[16] M. Granados-Cruz, Y. Shmaliy, C. K. Ahn, and S. Zhao, "Algorithmic innovations in extended unbiased fir filtering of nonlinear models," in Proc. 2015 Sci. Intormat. Conf. (SAI), 2015.

[17] G. Lasser and C. F. Mecklenbruker, "Self-interference noise limitations of rfid readers," in 2015 IEEE Int. Conf. on RFID, 2015, pp. 145-150.

[18] C. Jing, T. Sun, Q. Chen, M. Du, S. Wang, and J. Wang, "A robust noise mitigation method for the mobile rfid location in built environment," Sensors, vol. 19, pp. 1-16, 052019.

[19] C. J. D. Kurt E. Sundstrom, Paul Dietrich and A. Friefeld, "Rfid readers mitigating colored noise," Mar 2013.

[20] A. Bryson and D. Johansen, "Linear filtering for time-varying systems using measurements containing colored noise," IEEE Trans. Autom. Contr., vol. 10, no. 1, pp. 4-10, 1965.

[21] A. E. Bryson and L. J. Henrikson, "Estimation using sampled data containing sequentially correlated noise." J. Spacecraft Rockets, vol. 5 , no. 6 , pp. $662-665,1968$.

[22] M. G. Petovello, K. O'Keefe, G. Lachapelle, and M. E. Cannon, "Consideration of time-correlated errors in a Kalman filter applicable to GNSS," Journal of Geodesy, vol. 83, no. 1, pp. 51-56, Jan. 2009.

[23] Y. Shmaliy, S. Zhao, and C. K. Ahn, "Optimal and unbiased filtering with colored process noise using state differencing," IEEE Signal Process. Lett., vol. 26, no. 4, pp. 548-551, 2019.

[24] Z. Zhou, J. Wu, Y. Li, C. Fu, and H. Fourati, "Critical issues on kalman filter with colored and correlated system noises," Asian J. Control, vol. 19, no. 6, p. 1905-1919, 2017.

[25] F. Ding, D. Meng, J. Dai, Q. Li, A. Alsaedi, and T. Hayat, "Least squares based iterative parameter estimation algorithm for stochastic dynamical systems with ARMA noise using the model equivalence," Int. J. Control, Automat. Syst., vol. 16, no. 2, pp. 630-639, 2018.

[26] Y. S. Shmaliy, S. Zhao, and C. K. Ahn, "Kalman and UFIR state estimation with colored measurement noise using backward Euler method," IET Signal Process., vol. 14, no. 2, pp. 64-71, 2020.

[27] Y. S. Shmaliy, "An iterative Kalman-like algorithm ignoring noise and initial conditions," IEEE Trans. Signal Process., vol. 59, no. 6, pp. 2465-2473, 2011.
[28] F. Ramirez-Echeverria, A. Sarr, and Y. Shmaliy, "Optimal memory for discrete-time fir filters in state-space," IEEE Trans. Signal Process., vol. 62, pp. 557-561, 2014.

[29] P. Vorst, A. Koch, and A. Zell, "Efficient self-adjusting, similaritybased location fingerprinting with passive uhf rfid," in 2011 IEEE Int. Conf. on RFID-Techn. Appl., 2011, pp. 160-167.

[30] H. Ma, Y. Wang, K. Wang, and Z. Ma, "The optimization for hyperbolic positioning of uhf passive rfid tags," IEEE Trans. Automat. Sci. Eng., vol. 14, no. 4, pp. 1590-1600, 2017.

[31] Y. Gong, M. Shen, J. Zhang, O. Kaynak, W. Chen, and Z. Zhan, "Optimizing rfid network planning by using a particle swarm optimization algorithm with redundant reader elimination," IEEE Trans. Ind. Informat., vol. 8, no. 4, pp. 900-912, 2012.

[32] B. And, S. Baglio, V. Marletta, R. Crispino, and A. Pistorio, "A measurement strategy to assess the optimal design of an rfid-based navigation aid," IEEE Trans. Instrum. Meas., vol. 68, no. 7, pp. 23562362, 2019.

\section{Creative Commons Attribution License 4.0 (Attribution 4.0 International, CC BY 4.0)}

This article is published under the terms of the Creative Commons Attribution License 4.0 https://creativecommons.org/licenses/by/4.0/deed.en US 